\title{
THE JAMES CONSTANT OF NORMALIZED NORMS ON $\mathbb{R}^{2}$
}

WEERAYUTH NILSRAKOO AND SATIT SAEJUNG

Received 28 June 2005; Accepted 13 September 2005

We introduce a new class of normalized norms on $\mathbb{R}^{2}$ which properly contains all absolute normalized norms. We also give a criterion for deciding whether a given norm in this class is uniformly nonsquare. Moreover, an estimate for the James constant is presented and the exact value of some certain norms is computed. This gives a partial answer to the question raised by Kato et al.

Copyright (c) 2006 W. Nilsrakoo and S. Saejung. This is an open access article distributed under the Creative Commons Attribution License, which permits unrestricted use, distribution, and reproduction in any medium, provided the original work is properly cited.

\section{Introduction and preliminaries}

A norm $\|\cdot\|$ on $\mathbb{C}^{2}$ (resp., $\mathbb{R}^{2}$ ) is said to be absolute if $\|(z, w)\|=\|(|z|,|w|)\|$ for all $z, w \in \mathbb{C}($ resp., $\mathbb{R})$, and normalized if $\|(1,0)\|=\|(0,1)\|=1$. The $\ell_{p}$-norms $\|\cdot\|_{p}$ are such examples:

$$
\|(z, w)\|_{p}= \begin{cases}\left(|z|^{p}+|w|^{p}\right)^{1 / p} & \text { if } 1 \leq p<\infty \\ \max \{|z|,|w|\} & \text { if } p=\infty\end{cases}
$$

Let $A N_{2}$ be the family of all absolute normalized norms on $\mathbb{C}^{2}$ (resp., $\mathbb{R}^{2}$ ), and $\Psi_{2}$ the family of all continuous convex functions $\psi$ on $[0,1]$ such that $\psi(0)=\psi(1)=1$ and $\max \{1-t, t\} \leq \psi(t) \leq 1(0 \leq t \leq 1)$. According to Bonsall and Duncan [1], $A N_{2}$ and $\Psi_{2}$ are in a one-to-one correspondence under the equation

$$
\psi(t)=\|(1-t, t)\| \quad(0 \leq t \leq 1) .
$$

Indeed, for all $\psi \in \Psi_{2}$, let

$$
\|(z, w)\|_{\psi}= \begin{cases}(|z|+|w|) \psi\left(\frac{|w|}{|z|+|w|}\right) & \text { if }(z, w) \neq(0,0) \\ 0 & \text { if }(z, w)=(0,0)\end{cases}
$$

Hindawi Publishing Corporation Journal of Inequalities and Applications Volume 2006, Article ID 26265, Pages 1-12 DOI 10.1155/JIA/2006/26265 
Then $\|\cdot\|_{\psi} \in A N_{2}$, and $\|\cdot\|_{\psi}$ satisfies (1.2). From this result, we can consider many non- $\ell_{p}$-type norms easily. Now let

$$
\psi_{p}(t)= \begin{cases}\left((1-t)^{p}+t^{p}\right)^{1 / p} & \text { if } 1 \leq p<\infty, \\ \max \{1-t, t\} & \text { if } p=\infty\end{cases}
$$

Then $\psi_{p}(t) \in \Psi_{2}$ and, as is easily seen, the $\ell_{p}$-norm $\|\cdot\|_{p}$ is associated with $\psi_{p}$.

If $X$ is a Banach space, then $X$ is uniformly nonsquare if there exists $\delta \in(0,1)$ such that for any $x, y \in S_{X}$,

$$
\text { either }\|x+y\| \leq 2(1-\delta) \quad \text { or } \quad\|x-y\| \leq 2(1-\delta) \text {, }
$$

where $S_{X}=\{x \in X:\|x\|=1\}$. The James constant $J(X)$ is defined by

$$
J(X)=\sup \left\{\min \{\|x+y\|,\|x-y\|\}: x, y \in S_{X}\right\} .
$$

The modulus of convexity of $X, \delta_{X}:[0,2] \rightarrow[0,1]$ is defined by

$$
\delta_{X}(\varepsilon)=\inf \left\{1-\frac{1}{2}\|x+y\|: x, y \in S_{X},\|x-y\| \geq \varepsilon\right\} .
$$

The preceding parameters have been recently studied by several authors (cf. [4-6, 8, 9]). We collect together some known results.

Proposition 1.1. Let $X$ be a nontrivial Banach space, then

(i) $\sqrt{2} \leq J(X) \leq 2$ (Gao and Lau [5]),

(ii) if $X$ is a Hilbert space, then $J(X)=\sqrt{2}$; the converse is not true (Gao and Lau [5]),

(iii) $X$ is uniformly nonsquare if and only if $J(X)<2$ (Gao and Lau [5]),

(iv) $2 J(X)-2 \leq J\left(X^{*}\right) \leq J(X) / 2+1, J\left(X^{* *}\right)=J(X)$, and there exists a Banach space $X$ such that $J\left(X^{*}\right) \neq J(X)$ (Kato et al. [8]),

(v) if $2 \leq p \leq \infty$, then $\delta_{\ell_{p}}(\varepsilon)=1-\left(1-(\varepsilon / 2)^{p}\right)^{1 / p}$ (Hanner [6]),

(vi) $J(X)=\sup \left\{\varepsilon \in(0,2): \delta_{X}(\varepsilon) \leq 1-\varepsilon / 2\right\}$ (Gao and Lau [5]).

The paper is organized as follows. In Section 2 we introduce a new class of normalized norms on $\mathbb{R}^{2}$. This class properly contains all absolute normalized norms of Bonsall and Duncan [1]. The so-called generalized Day-James space, $\ell_{\psi}-\ell_{\varphi}$, where $\psi, \varphi \in \Psi_{2}$, is introduced and studied. More precisely, we prove that $\left(\ell_{\psi}-\ell_{\varphi}\right)^{*}=\ell_{\psi^{*}}-\ell_{\varphi^{*}}$ where $\psi^{*}$ and $\varphi^{*}$ are the dual functions of $\psi$ and $\varphi$, respectively. In Section 3, the upper bound of the James constant of the generalized Day-James space is given. Furthermore, we compute $J\left(\ell_{\psi}-\ell_{\infty}\right)$ and deduce that every generalized Day-James space except $\ell_{1}-\ell_{1}$ and $\ell_{\infty}-\ell_{\infty}$ is uniformly nonsquare. This result strengthens Corollary 3 of Saito et al. [10].

\section{Generalized Day-James spaces}

In this section, we introduce a new class of normalized norms on $\mathbb{R}^{2}$ which properly contains all absolute normalized norms of Bonsall and Duncan [1]. Moreover, we introduce a two-dimensional normed space which is a generalization of Day-James $\ell_{p}-\ell_{q}$ spaces. 
Lemma 2.1. Let $\psi \in \Psi_{2}$ and let $\|\cdot\|_{\psi, \psi_{\infty}}$ be a function on $\mathbb{R}^{2}$ defined by, for all $(z, w) \in \mathbb{R}^{2}$,

$$
\begin{aligned}
\|(z, w)\|_{\psi, \psi_{\infty}} & :=\max \left\{\left\|\left(z^{+}, w^{+}\right)\right\|_{\psi},\left\|\left(z^{-}, w^{-}\right)\right\|_{\psi}\right\}, \\
& = \begin{cases}\|(z, w)\|_{\psi} & \text { if } z w \geq 0, \\
\|(z, w)\|_{\infty} & \text { if } z w \leq 0,\end{cases}
\end{aligned}
$$

where $x^{+}$and $x^{-}$are positive and negative parts of $x \in \mathbb{R}$, that is, $x^{+}=\max \{x, 0\}$ and $x^{-}=$ $\max \{-x, 0\}$. Then $\|\cdot\|_{\psi, \psi_{\infty}}$ is a norm on $\mathbb{R}^{2}$.

For convenience, we put $\mathscr{B}_{\psi_{1}, \psi_{2}}:=\left\{(z, w) \in \mathbb{R}^{2}:\|(z, w)\|_{\psi_{1}, \psi_{2}} \leq 1\right\}$.

Theorem 2.2. Let $\psi, \varphi \in \Psi_{2}$ and

$$
\|(z, w)\|_{\psi, \varphi}:= \begin{cases}\|(z, w)\|_{\psi} & \text { if } z w \geq 0 \\ \|(z, w)\|_{\varphi} & \text { if } z w \leq 0\end{cases}
$$

for all $(z, w) \in \mathbb{R}^{2}$. Then $\|\cdot\|_{\psi, \varphi}$ is a norm on $\mathbb{R}^{2}$. Denote by $N_{2}$ the family of all such preceding norms.

Proof. Let $\psi, \varphi \in \Psi_{2}$, we only show $\|\cdot\|_{\psi, \varphi}$ satisfies the triangle inequality. To this end, it suffices to prove that $\mathscr{B}_{\psi, \varphi}$ is convex. By Lemma 2.1 , we have that $\mathscr{B}_{\psi, \psi_{\infty}}$ and $\mathscr{B}_{\varphi, \psi_{\infty}}$ are closed unit balls of $\|\cdot\|_{\psi, \psi_{\infty}}$ and $\|\cdot\|_{\varphi, \psi_{\infty}}$, respectively, and so $\mathscr{B}_{\psi, \psi_{\infty}}$ and $\mathscr{B}_{\varphi, \psi_{\infty}}$ are convex sets. We define $T: \mathbb{R}^{2} \rightarrow \mathbb{R}^{2}$ by

$$
T((z, w))=(-z, w) \quad \forall(z, w) \in \mathbb{R}^{2} .
$$

Then $T$ is a linear operator and $T\left(\mathscr{B}_{\varphi, \psi_{\infty}}\right)=\mathscr{B}_{\psi_{\infty}, \varphi}$, which implies that $\mathscr{B}_{\psi_{\infty}, \varphi}$ is convex and so $\mathscr{B}_{\psi, \varphi}=\mathscr{S}_{\psi_{\infty}, \varphi} \cap \mathscr{S}_{\psi, \psi_{\infty}}$ is convex.

Taking $\psi=\psi_{p}$ and $\varphi=\psi_{q}(1 \leq p, q \leq \infty)$ in Theorem 2.2, we obtain the following.

Corollary 2.3 (Day-James $\ell_{p}-\ell_{q}$ spaces). For $1 \leq p, q \leq \infty$, denote by $\ell_{p}-\ell_{q}$ the DayJames space, that is, $\mathbb{R}^{2}$ with the norm defined by, for all $(z, w) \in \mathbb{R}^{2}$,

$$
\|(z, w)\|_{p, q}= \begin{cases}\|(z, w)\|_{p} & \text { if } z w \geq 0 \\ \|(z, w)\|_{q} & \text { if } z w \leq 0\end{cases}
$$

James [7] considered the $\ell_{p}-\ell_{p^{\prime}}$ space as an example of a Banach space which is isometric to its dual but which is not given by a Hilbert norm when $p \neq 2$. Day [2] considered even more general spaces, namely, if $(X,\|\cdot\|)$ is a two-dimensional Banach space and $\left(X^{*},\|\cdot\|^{*}\right)$ its dual, then the $X-X^{*}$ space is the space $X$ with the norm defined by, for all $(z, w) \in \mathbb{R}^{2}$,

$$
\|(z, w)\|_{X, X^{*}}= \begin{cases}\|(z, w)\| & \text { if } z w \geq 0 \\ \|(z, w)\|^{*} & \text { if } z w \leq 0\end{cases}
$$




\section{The James constant of normalized norms on $\mathbb{R}^{2}$}

For $\psi, \varphi \in \Psi_{2}$, denote by $\ell_{\psi}-\ell_{\varphi}$ the generalized Day-James space, that is, $\mathbb{R}^{2}$ with the norm $\|\cdot\|_{\psi, \varphi}$ defined by (2.2). For $\psi_{p}$ defined by (1.4), we write $\ell_{\psi}-\ell_{p}$ for $\ell_{\psi}-\ell_{\psi_{p}}$. For example, if $1 \leq p, q \leq \infty, \ell_{p}-\ell_{q}$ means $\ell_{\psi_{p}}-\ell_{\psi_{q}}$.

It is worthwhile to mention that there is a normalized norm which is not absolute.

Proposition 2.4. There is $\psi \in \Psi_{2}$ such that $\ell_{\psi}-\ell_{\infty}$ is not isometrically isomorphic to $\ell_{\varphi}-\ell_{\varphi}$ for all $\varphi \in \Psi_{2}$.

Proof. Let

$$
\psi(t):= \begin{cases}1-t & \text { if } 0 \leq t \leq \frac{1}{8} \\ \frac{11-4 t}{12} & \text { if } \frac{1}{8} \leq t \leq \frac{1}{2} \\ \frac{1+t}{2} & \text { if } \frac{1}{2} \leq t \leq 1 .\end{cases}
$$

We observe that the sphere of $\ell_{\psi}-\ell_{\infty}$ is the octagon whose right half consists of 4 segments of different lengths. Suppose that there are $\varphi \in \Psi_{2}$ and an isometric isomorphism from $\ell_{\psi}-\ell_{\infty}$ onto $\ell_{\varphi}-\ell_{\varphi}$. Since the image of each segment in $\ell_{\psi}-\ell_{\infty}$ is again a segment of the same length in $\ell_{\varphi}-\ell_{\varphi}$, the sphere of $\ell_{\varphi}-\ell_{\varphi}$ must be the octagon whose each corresponding side has the same length (measured by $\|\cdot\|_{\varphi}$ ). We show that this cannot happen. Consider $(1,0) \in S_{\ell_{\varphi}-\ell_{\varphi}}$. If $(1,0)$ is an extreme point of $B_{\ell_{\varphi}-\ell_{\varphi}}$, then $S_{\ell_{\varphi}-\ell_{\varphi}}$ contains 4 segments of same lengths since $\|\cdot\|_{\varphi}$ is absolute. On the other hand, if $(1,0)$ is an not extreme point of $B_{\ell_{\varphi}-\ell_{\varphi}}$, again $S_{\ell_{\varphi}-\ell_{\varphi}}$ contains 4 segments of same lengths.

Next, we prove that the dual of a generalized Day-James space is again a generalized Day-James space. Recall that, for $\psi \in \Psi_{2}$, the dual function $\psi^{*}$ of $\psi$ is defined by

$$
\psi^{*}(s)=\max _{0 \leq t \leq 1} \frac{(1-s)(1-t)+s t}{\psi(t)}
$$

for all $s \in[0,1]$. It was proved that $\psi^{*} \in \Psi_{2}$ and $\left(\ell_{\psi}-\ell_{\psi}\right)^{*}=\ell_{\psi^{*}-} \ell_{\psi^{*}}$ (see $[3$, Proposition 1 and Theorem 2]). We generalize this result to our spaces as follows.

Theorem 2.5. For $\psi, \varphi \in \Psi_{2}$, there is an isometric isomorphism that identifies $\left(\ell_{\psi}-\ell_{\varphi}\right) *$ with $\ell_{\psi^{*}}-\ell_{\varphi^{*}}$ such that if $f \in\left(\ell_{\psi^{-}}-\ell_{\varphi}\right) *$ is identified with the element $(z, w) \in \ell_{\psi^{*}}-\ell_{\varphi^{*}}$, then

$$
f(u, v)=z u+w v
$$

for all $(u, v) \in \mathbb{R}^{2}$.

Proof. We can prove analogous to [3, Theorem 2].

\section{The James constant and uniform nonsquareness}

The next lemmas are crucial for proving the main theorems.

Lemma 3.1. Let $\psi, \varphi \in \Psi_{2}$. Then

(i) $\|\cdot\|_{\infty} \leq\|\cdot\|_{\psi, \varphi} \leq\|\cdot\|_{1}$, 
(ii) $\left(1 / M_{\psi, \varphi}\right)\|\cdot\|_{\psi} \leq\|\cdot\|_{\psi, \varphi} \leq M_{\varphi, \psi}\|\cdot\|_{\psi}$,

(iii) $\left(1 / M_{\varphi, \psi}\right)\|\cdot\|_{\varphi} \leq\|\cdot\|_{\psi, \varphi} \leq M_{\psi, \varphi}\|\cdot\|_{\varphi}$, where $M_{\varphi, \psi}=\max _{0 \leq t \leq 1} \varphi(t) / \psi(t)$ and $M_{\psi, \varphi}=\max _{0 \leq t \leq 1} \psi(t) / \varphi(t)$.

Lemma 3.2. Let $\psi, \varphi \in \Psi_{2}$ and let $Q_{i}(i=1, \ldots, 4)$ denote the ith quadrant in $\mathbb{R}^{2}$. Suppose that $x, y \in S_{\ell_{\psi}-\ell_{\varphi}}$, then the following statements are true.

(i) If $x, y \in Q_{1}$, then $x+y \in Q_{1}$ and $x-y \in Q_{2} \cup Q_{4}$.

(ii) If $x, y \in Q_{2}$, then $x+y \in Q_{2}$ and $x-y \in Q_{1} \cup Q_{3}$.

(iii) If $\psi(t) \leq \varphi(t)$ for all $t \in[0,1]$ and $x-y \in Q_{2}^{\circ} \cup Q_{4}^{\circ}$, where $Q_{2}^{\circ}$ and $Q_{4}^{\circ}$ are the interiors of $Q_{2}$ and $Q_{4}$, respectively, then $x+y \in Q_{1} \cup Q_{3}$.

We will estimate the James constant of $\ell_{\psi}-\ell_{\varphi}$.

Theorem 3.3. Let $\psi, \varphi \in \Psi_{2}$ with $\psi(t) \leq \varphi(t)$ for all $t \in[0,1]$, let $M_{\varphi, \psi}=\max _{0 \leq t \leq 1} \varphi(t) / \psi(t)$, and let $\delta_{\psi}(\cdot)$ be the modulus of convexity of $\ell_{\psi}-\ell_{\psi}$. Then for $\varepsilon \in[0,2]$,

$$
\delta_{\psi, \varphi}(\varepsilon) \geq \min \left\{1-M_{\varphi, \psi}\left(1-\delta_{\psi}(\varepsilon)\right), \delta_{\psi}\left(\frac{\varepsilon}{M_{\varphi, \psi}}\right)\right\}
$$

where $\delta_{\psi, \varphi}(\cdot)$ is the modulus of convexity of $\ell_{\psi}-\ell_{\varphi}$. Consequently,

$$
J\left(\ell_{\psi}-\ell_{\varphi}\right) \leq \sup \left\{\varepsilon \in(0,2): \varepsilon \leq 2 M_{\varphi, \psi}\left(1-\delta_{\psi}(\varepsilon)\right) \text { or } \varepsilon \leq 2\left(1-\delta_{\psi}\left(\frac{\varepsilon}{M_{\varphi, \psi}}\right)\right)\right\} .
$$

Proof. By Lemma 3.1(ii), we have

$$
\|\cdot\|_{\psi} \leq\|\cdot\|_{\psi, \varphi} \leq M_{\varphi, \psi}\|\cdot\|_{\psi}
$$

We now evaluate the modulus of convexity $\delta_{\psi, \varphi}$ for $\ell_{\psi}-\ell_{\varphi}$. We consider two cases.

Case 1. Take $\|x\|_{\psi, \varphi}=\|y\|_{\psi, \varphi}=1$ with $\|x-y\|_{\psi, \varphi} \geq \varepsilon$, where $x-y \in Q_{1} \cup Q_{3}$. Thus $\|x\|_{\psi} \leq 1,\|y\|_{\psi} \leq 1$, and $\|x-y\|_{\psi} \geq \varepsilon$, which implies that

$$
\frac{1}{2}\|x+y\|_{\psi} \leq 1-\delta_{\psi}(\varepsilon)
$$

This in turn implies

$$
\frac{1}{2}\|x+y\|_{\psi, \varphi} \leq \frac{1}{2} M_{\varphi, \psi}\|x+y\|_{\psi} \leq M_{\varphi, \psi}\left(1-\delta_{\psi}(\varepsilon)\right)
$$

thus

$$
1-\frac{1}{2}\|x+y\|_{\psi, \varphi} \geq 1-M_{\varphi, \psi}\left(1-\delta_{\psi}(\varepsilon)\right)
$$

Case 2. Now take $x, y$ as above, but with $x-y \in Q_{2}^{\circ} \cup Q_{4}^{\circ}$. By Lemma 3.2(iii), $x+y \in$ $Q_{1} \cup Q_{3}$. Since $\|x-y\|_{\psi, \varphi} \geq \varepsilon$,

$$
\|x-y\|_{\psi} \geq \frac{\|x-y\|_{\psi, \varphi}}{M_{\varphi, \psi}} \geq \frac{\varepsilon}{M_{\varphi, \psi}} .
$$


6 The James constant of normalized norms on $\mathbb{R}^{2}$

Then

$$
\frac{1}{2}\|x+y\|_{\psi, \varphi}=\frac{1}{2}\|x+y\|_{\psi} \leq 1-\delta_{\psi}\left(\frac{\varepsilon}{M_{\varphi, \psi}}\right)
$$

and so

$$
1-\frac{1}{2}\|x+y\|_{\psi, \varphi} \geq \delta_{\psi}\left(\frac{\varepsilon}{M_{\varphi, \psi}}\right)
$$

Hence we obtain (3.1). By Proposition 1.1(vi), (3.2) follows.

The following corollary shows that we can have equality in (3.2).

Corollary $3.4[4,8]$. If $1 \leq q \leq p<\infty$ and $p \geq 2$, then

$$
J\left(\ell_{p}-\ell_{q}\right) \leq 2\left(\frac{2^{p / q}}{2^{p / q}+2}\right)^{1 / p} .
$$

In particular, if $p=2$ and $q=1$, then $J\left(\ell_{2}-\ell_{1}\right)=\sqrt{8 / 3}$.

Proof. It follows that since

$$
M_{\psi_{q}, \psi_{p}}=2^{1 / q-1 / p}, \quad \delta_{\ell_{p}-\ell_{p}}(\varepsilon)=1-\left(1-\left(\frac{\varepsilon}{2}\right)^{p}\right)^{1 / p}
$$

Moreover, if $p=2$ and $q=1$, then $J\left(\ell_{2}-\ell_{1}\right) \leq \sqrt{8 / 3}$. Now we put

$$
x_{0}=\left(\frac{2+\sqrt{2}}{2 \sqrt{3}}, \frac{2-\sqrt{2}}{2 \sqrt{3}}\right), \quad y_{0}=\left(\frac{2-\sqrt{2}}{2 \sqrt{3}}, \frac{2+\sqrt{2}}{2 \sqrt{3}}\right) .
$$

Then

$$
\left\|x_{0}\right\|_{2,1}=\left\|y_{0}\right\|_{2,1}=1, \quad\left\|x_{0} \pm y_{0}\right\|_{2,1}=\sqrt{\frac{8}{3}} .
$$

Theorem 3.5. Let $\psi, \varphi \in \Psi_{2}$ with $\psi(t) \leq \varphi(t)$ for all $t \in[0,1]$, let $M_{\varphi, \psi}=\max _{0 \leq t \leq 1} \varphi(t) / \psi(t)$, and let $\delta_{\varphi}(\cdot)$ be the modulus of convexity of $\ell_{\varphi}-\ell_{\varphi}$. Then for $\varepsilon \in[0,2]$,

$$
\delta_{\psi, \varphi}(\varepsilon) \geq 1-M_{\varphi, \psi}\left(1-\delta_{\varphi}\left(\frac{\varepsilon}{M_{\varphi, \psi}}\right)\right),
$$

where $\delta_{\psi, \varphi}(\cdot)$ is the modulus of convexity of $\ell_{\psi}-\ell_{\varphi}$. Consequently,

$$
J\left(\ell_{\psi}-\ell_{\varphi}\right) \leq \sup \left\{\varepsilon \in(0,2): \varepsilon \leq 2 M_{\varphi, \psi}\left(1-\delta_{\varphi}\left(\frac{\varepsilon}{M_{\varphi, \psi}}\right)\right)\right\} .
$$

Proof. By Lemma 3.1(iii), we have

$$
\frac{1}{M_{\varphi, \psi}}\|\cdot\|_{\varphi} \leq\|\cdot\|_{\psi, \varphi} \leq\|\cdot\|_{\varphi} .
$$


We now evaluate the modulus of convexity $\delta_{\psi, \varphi}$ for $\ell_{\psi}-\ell_{\varphi}$. Let

$$
\|x\|_{\psi, \varphi}=\|y\|_{\psi, \varphi}=1 \quad \text { with }\|x-y\|_{\psi, \varphi} \geq \varepsilon .
$$

Then

$$
\begin{aligned}
\frac{1}{M_{\varphi, \psi}}\|x\|_{\varphi} & \leq 1, \quad \frac{1}{M_{\varphi, \psi}}\|y\|_{\varphi} \leq 1, \\
\frac{1}{M_{\varphi, \psi}}\|x-y\|_{\varphi} & \geq \frac{1}{M_{\varphi, \psi}}\|x-y\|_{\psi, \varphi} \geq \frac{\varepsilon}{M_{\varphi, \psi}},
\end{aligned}
$$

which implies that

$$
\frac{1}{2 M_{\varphi, \psi}}\|x+y\|_{\varphi} \leq 1-\delta_{\varphi}\left(\frac{\varepsilon}{M_{\varphi, \psi}}\right)
$$

This in turn implies that

$$
\frac{1}{2 M_{\varphi, \psi}}\|x+y\|_{\psi, \varphi} \leq \frac{1}{2 M_{\varphi, \psi}}\|x+y\|_{\varphi} \leq 1-\delta_{\varphi}\left(\frac{\varepsilon}{M_{\varphi, \psi}}\right),
$$

thus

$$
1-\frac{1}{2}\|x+y\|_{\psi, \varphi} \geq 1-M_{\varphi, \psi}\left(1-\delta_{\varphi}\left(\frac{\varepsilon}{M_{\varphi, \psi}}\right)\right)
$$

Hence we obtain (3.14). By Proposition 1.1(vi), (3.15) follows.

Corollary 3.6. If $2 \leq q \leq p<\infty$, then

$$
J\left(\ell_{p}-\ell_{q}\right) \leq 2^{1-1 / p} .
$$

It is easy to see that the estimate (3.22) is better than one obtained in [4, Example $2.4(3)]$.

For some generalized Day-James spaces, [8, Corollary 4] of Kato et al. gives only rough result for the estimate of the James constant, that is, for $\psi \in \Psi_{2}$,

$$
\frac{2}{M} \leq J\left(\ell_{\psi}-\ell_{\infty}\right) \leq 2 M
$$

where $M=\max _{0 \leq t \leq 1} \psi_{\infty}(t) / \psi(t)$.

However, the following theorem gives the exact value of the James constant of these spaces.

Theorem 3.7. Let $\psi \in \Psi_{2}$. Then

$$
J\left(\ell_{\psi}-\ell_{\infty}\right)=1+\frac{1 / 2}{\psi(1 / 2)}
$$


8 The James constant of normalized norms on $\mathbb{R}^{2}$

Proof. For our convenience, we write $\|\cdot\|$ instead of $\|\cdot\|_{\psi, \psi_{\infty}}$. Let $x, y \in S_{\ell_{\psi}-\ell_{\infty}}$. We prove that

$$
\text { either }\|x+y\| \leq 1+\frac{1 / 2}{\psi(1 / 2)} \quad \text { or } \quad\|x-y\| \leq 1+\frac{1 / 2}{\psi(1 / 2)} \text {. }
$$

Let us consider the following cases.

Case 1. $x, y \in Q_{1}$. Let $x=(a, b)$ and $y=(c, d)$ where $a, b, c, d \in[0,1]$. By Lemma 3.2(i), we have $x-y \in Q_{2} \cup Q_{4}$. Then

$$
\|x-y\|=\max \{|a-c|,|b-d|\} \leq 1 \leq 1+\frac{1 / 2}{\psi(1 / 2)}
$$

Case 2. $x, y \in Q_{2}$. If $x, y$ lies in the same segment, then $\|x-y\| \leq 1$. We now suppose that $x=(-1, a)$ and $y=(-c, 1)$ where $a, c \in[0,1]$.

Subcase 2.1. $a \leq(1 / 2) / \psi(1 / 2)$ and $c \leq(1 / 2) / \psi(1 / 2)$. Then

$$
\|x+y\|=\|(-1-c, 1+a)\|_{\infty}=\max \{1+c, 1+a\} \leq 1+\frac{1 / 2}{\psi(1 / 2)} .
$$

Subcase 2.2. $a \geq(1 / 2) / \psi(1 / 2)$ or $c \geq(1 / 2) / \psi(1 / 2)$. Put $z=(-1,1)$, then

$$
\|x-y\| \leq\|x-z\|+\|z-y\|=1-a+1-c \leq 1+1-\frac{1 / 2}{\psi(1 / 2)} \leq 1+\frac{1 / 2}{\psi(1 / 2)}
$$

From now on, we may assume without loss of generality that there is $\beta \in[1 / 2,1]$ such that $\psi(\beta) \leq \psi(t)$ for all $t \in[0,1]$. Indeed, $J\left(\ell_{\psi}-\ell_{\infty}\right)=J\left(\ell_{\tilde{\psi}}-\ell_{\infty}\right)$ where $\tilde{\psi}(t)=\psi(1-t)$ for all $t \in[0,1]$.

Case 3. $x \in Q_{1}$ and $y \in Q_{2}$. Let $x=(a, b), y=(-c, 1)$ where $a, b, c \in[0,1]$. We consider three subcases.

Subcase 3.1. $a \leq(1 / 2) / \psi(1 / 2)$ or $c \leq(1 / 2) / \psi(1 / 2)$. Then

$$
\|x-y\|=\|(a+c, b-1)\|_{\infty}=\max \{a+c, 1-b\} \leq 1+\frac{1 / 2}{\psi(1 / 2)} .
$$

Subcase 3.2. $(1 / 2) / \psi(1 / 2) \leq a \leq c$. Then $b \leq(1 / 2) / \psi(1 / 2)$ and

$$
\|x+y\|=\|(a-c, b+1)\|_{\infty}=\max \{c-a, 1+b\} \leq 1+\frac{1 / 2}{\psi(1 / 2)} .
$$

Subcase 3.3. $(1 / 2) / \psi(1 / 2)<c \leq a$. We write $a=\left(1-t_{0}\right) / \psi\left(t_{0}\right), b=t_{0} / \psi\left(t_{0}\right)$ where $t_{0}=$ $b /(a+b)$ and $0 \leq t_{0} \leq 1 / 2$. By the convexity of $\psi$ and $\psi(t) \geq \psi(\beta)$ for all $0 \leq t \leq 1$, we 
have $\psi\left(t_{0}\right) \geq \psi(1 / 2)$ and so $1 / \psi\left(t_{0}\right) \leq 1 / \psi(1 / 2)$. By Lemma 3.1(i),

$$
\begin{aligned}
\|x+y\| & =\|(a, b)+(-c, 1)\| \leq\|(a-c, b+1)\|_{1} \\
& =a-c+b+1=\frac{1}{\psi\left(t_{0}\right)}+1-c \\
& \leq \frac{1}{\psi(1 / 2)}+1-\frac{1 / 2}{\psi(1 / 2)}=1+\frac{1 / 2}{\psi(1 / 2)} .
\end{aligned}
$$

Case 4. $x \in Q_{1}$ and $y \in Q_{2}$. Let $x=(a, b), y=(-1, c)$ where $a, b, c \in[0,1]$. We consider three subcases.

Subcase 4.1. $b \leq(1 / 2) / \psi(1 / 2)$ or $c \leq(1 / 2) / \psi(1 / 2)$. Then

$$
\|x+y\|=\|(a-1, b+c)\|_{\infty}=\max \{1-a, b+c\} \leq 1+\frac{1 / 2}{\psi(1 / 2)} .
$$

Subcase 4.2. $(1 / 2) / \psi(1 / 2)<b \leq c$. Then $a \leq(1 / 2) / \psi(1 / 2)$ and

$$
\|x-y\|=\|(1+a, b-c)\|_{\infty}=\max \{1+a, c-b\} \leq 1+\frac{1 / 2}{\psi(1 / 2)} .
$$

Subcase 4.3. $(1 / 2) / \psi(1 / 2)<c \leq b$. We write $a=\left(1-t_{0}\right) / \psi\left(t_{0}\right), b=t_{0} / \psi\left(t_{0}\right)$, where $t_{0}=$ $b /(a+b)$ and $1 / 2 \leq t_{0} \leq 1$. We choose $\alpha=b /(a+2 b-1)$, then

$$
\frac{1}{2} \leq \alpha \leq 1, \quad a=\frac{1-2 \alpha}{\alpha} b+1
$$

Since $b-c \leq 1+a$ and $b \leq 1$,

$$
\frac{b-c}{1+a+b-c} \leq \frac{1}{2} \leq t_{0} \leq \alpha
$$

Let

$$
\psi_{\alpha}(t)= \begin{cases}\frac{\alpha-1}{\alpha} t+1 & \text { if } 0 \leq t \leq \alpha \\ t & \text { if } \alpha \leq t \leq 1\end{cases}
$$

We see that $\psi_{\alpha}\left(t_{0}\right)=\psi\left(t_{0}\right)$. By the convexity of $\psi$, we have

$$
\psi(t) \leq \psi_{\alpha}(t) \quad \forall t \leq t_{0}
$$


10 The James constant of normalized norms on $\mathbb{R}^{2}$

Therefore,

$$
\begin{aligned}
\|x-y\| & =\|(a+1, b-c)\|_{\psi}=(1+a+b-c) \psi\left(\frac{b-c}{1+a+b-c}\right) \\
& \leq(1+a+b-c) \psi_{\alpha}\left(\frac{b-c}{1+a+b-c}\right)=\frac{\alpha-1}{\alpha}(b-c)+1+a+b-c \\
& =1+a+\frac{2 \alpha-1}{\alpha} b-\frac{2 \alpha-1}{\alpha} c=1+1-\frac{2 \alpha-1}{\alpha} c \\
& <1+1-\frac{2 \alpha-1}{\alpha} \frac{1 / 2}{\psi(1 / 2)}=1+\frac{1 / 2}{\psi(1 / 2)}+1-\frac{3 \alpha-1}{2 \alpha} \frac{1}{\psi(1 / 2)} \\
& =1+\frac{1 / 2}{\psi(1 / 2)}+1-\frac{\psi_{\alpha}(1 / 2)}{\psi(1 / 2)} \leq 1+\frac{1 / 2}{\psi(1 / 2)} .
\end{aligned}
$$

Finally, we conclude that

$$
J\left(\ell_{\psi}-\ell_{\infty}\right) \leq 1+\frac{1 / 2}{\psi(1 / 2)}
$$

Now, we put $x_{0}=((1 / 2) / \psi(1 / 2),(1 / 2) / \psi(1 / 2))$ and $y_{0}=(-1,1)$, then

$$
\left\|x_{0}\right\|=\left\|y_{0}\right\|=1, \quad\left\|x_{0} \pm y_{0}\right\|=1+\frac{1 / 2}{\psi(1 / 2)} .
$$

Thus,

$$
J\left(\ell_{\psi}-\ell_{\infty}\right) \geq \min \left\{\left\|x_{0}-y_{0}\right\|,\left\|x_{0}+y_{0}\right\|\right\}=1+\frac{1 / 2}{\psi(1 / 2)} .
$$

This together with (3.39) completes the proof.

Corollary 3.8 [4, Example 2.4(2)]. Let $1 \leq p \leq \infty$, then

$$
J\left(\ell_{p}-\ell_{\infty}\right)=1+\left(\frac{1}{2}\right)^{1 / p} .
$$

Indeed, $\psi_{p}(1 / 2)=2^{1 / p-1}$.

We now obtain the bounds for $J\left(\ell_{\psi}-\ell_{1}\right)$.

Corollary 3.9. Let $\psi \in \Psi_{2}$. Then

$$
2 \min _{0 \leq t \leq 1} \psi(t) \leq J\left(\ell_{\psi}-\ell_{1}\right) \leq \frac{3}{2}+\frac{1}{2} \min _{0 \leq t \leq 1} \psi(t)
$$

Proof. Note that $\psi^{*}(1 / 2)=\max _{0 \leq t \leq 1}(1 / 2) / \psi(t)=1 / 2 \min _{0 \leq t \leq 1} \psi(t)$. By Theorem 3.7, we have $J\left(\ell_{\psi^{*}-} \ell_{\infty}\right)=1+\min _{0 \leq t \leq 1} \psi(t)$. Applying Proposition 1.1(iv), the assertion is obtained.

We now improve the upper bound for $J\left(\ell_{p}-\ell_{1}\right)$ (see also Corollary 3.4). 
Corollary 3.10. Let $1 \leq p<\infty$. Then

$$
J\left(\ell_{p}-\ell_{1}\right) \leq \frac{3}{2}+\left(\frac{1}{2}\right)^{2-1 / p} .
$$

In particular, if $p \geq 2$, then

$$
J\left(\ell_{p}-\ell_{1}\right) \leq \min \left\{\frac{4}{\left(2^{p}+2\right)^{1 / p}}, \frac{3}{2}+\left(\frac{1}{2}\right)^{2-1 / p}\right\} .
$$

The following corollary follows by Theorem 3.7 and Corollary 3.9.

Corollary 3.11. Let $\psi \in \Psi_{2}$. Then

(i) $\ell_{\psi}-\ell_{\infty}$ is uniformly nonsquare if and only if $\psi \neq \psi_{\infty}$,

(ii) $\ell_{\psi}-\ell_{1}$ is uniformly nonsquare if and only if $\psi \neq \psi_{1}$.

We can say more about the uniform nonsquareness of $\ell_{\psi}-\ell_{\varphi}$.

Theorem 3.12. Let $\psi, \varphi \in \Psi_{2}$. Then all $\ell_{\psi}-\ell_{\varphi}$ except $\ell_{1}-\ell_{1}$ and $\ell_{\infty}-\ell_{\infty}$ are uniformly nonsquare.

Proof. If $\psi=\varphi$, we are done by [10, Corollary 3]. Assume that $\psi \neq \varphi$. We prove that $\ell_{\psi}-\ell_{\varphi}$ is uniformly nonsquare. Suppose not, that is, there are $x, y \in S_{\ell_{\psi}-\ell_{\varphi}}$ such that $\|x \pm y\|_{\psi, \varphi}=$ 2. We consider three cases.

Case 1. $x, y \in Q_{1}$. Then

$$
\begin{aligned}
\|x\|_{\psi, 1} & =\|x\|_{\psi}=\|x\|_{\psi, \varphi}=1, \\
\|y\|_{\psi, 1} & =\|y\|_{\psi}=\|y\|_{\psi, \varphi}=1 .
\end{aligned}
$$

It follows by Lemma 3.2(i) that $x+y \in Q_{1}$ and $x-y \in Q_{2} \cup Q_{4}$. Therefore

$$
\begin{gathered}
\|x+y\|_{\psi, 1}=\|x+y\|_{\psi, \varphi}=2, \\
2=\|x-y\|_{\psi, \varphi} \leq\|x-y\|_{1}=\|x-y\|_{\psi, 1} \leq 2 .
\end{gathered}
$$

Hence $\|x \pm y\|_{\psi, 1}=2$ and this implies that $\ell_{\psi}-\ell_{1}$ is not uniformly nonsquare. By Corollary 3.11(ii), we have $\psi=\psi_{1}$. Again, since $\ell_{\psi}-\ell_{\varphi}=\ell_{1}-\ell_{\varphi}$ is not uniformly nonsquare, $\varphi=\psi_{1}=$ $\psi$; a contradiction.

Case 2. $x, y \in Q_{2}$. It is similar to Case 1 , so we omit the proof.

Case 3. $x:=(a, b) \in Q_{1}$ and $y:=(-c, d) \in Q_{2}$ where $a, b, c, d \in[0,1]$. Since $\|x+y\|_{\psi, \varphi}=2$, the line segment joining $x$ and $y$ must lie in the sphere. In particular, there is $\alpha \in[0,1]$ such that

$$
(0,1)=\alpha x+(1-\alpha) y
$$

It follows that $b=1$ since $b, d \leq 1$. Similarly consider $x$ and $-y$ instead of $x$ and $y$, we can also conclude that $a=1$. Hence $\|(1,1)\|_{\psi}=\|(1,1)\|_{\psi, \varphi}=1$, that is, $\psi(1 / 2)=1 / 2$. Then $\psi=\psi_{\infty}$ and so $\ell_{\psi}-\ell_{\varphi}=\ell_{\infty}-\ell_{\varphi}$ is not uniformly nonsquare. By Corollary 3.11(i), we have $\varphi=\psi_{\infty}=\psi ;$ a contradiction. 
12 The James constant of normalized norms on $\mathbb{R}^{2}$

\section{Acknowledgments}

The authors would like to thank the referee for suggestions which led to a presentation of the paper. The second author was supported by the Thailand Research Fund under Grant BRG 4780013.

\section{References}

[1] F. F. Bonsall and J. Duncan, Numerical Ranges, Vol. II, Cambridge University Press, New York, 1973.

[2] M. M. Day, Uniform convexity in factor and conjugate spaces, Annals of Mathematics. Second Series 45 (1944), no. 2, 375-385.

[3] S. Dhompongsa, A. Kaewkhao, and S. Saejung, Uniform smoothness and U-convexity of $\psi$-direct sums, Journal of Nonlinear and Convex Analysis 6 (2005), no. 2, 327-338.

[4] S. Dhompongsa, A. Kaewkhao, and S. Tasena, On a generalized James constant, Journal of Mathematical Analysis and Applications 285 (2003), no. 2, 419-435.

[5] J. Gao and K.-S. Lau, On the geometry of spheres in normed linear spaces, Journal of Australian Mathematical Society. Series A 48 (1990), no. 1, 101-112.

[6] O. Hanner, On the uniform convexity of $L^{p}$ and $l^{p}$, Arkiv för Matematik 3 (1956), 239-244.

[7] R. C. James, Inner product in normed linear spaces, Bulletin of the American Mathematical Society 53 (1947), 559-566.

[8] M. Kato, L. Maligranda, and Y. Takahashi, On James and Jordan-von Neumann constants and the normal structure coefficient of Banach spaces, Studia Mathematica 144 (2001), no. 3, 275-295.

[9] K.-I. Mitani and K.-S. Saito, The James constant of absolute norms on $\mathbb{R}^{2}$, Journal of Nonlinear and Convex Analysis 4 (2003), no. 3, 399-410.

[10] K.-S. Saito, M. Kato, and Y. Takahashi, Von Neumann-Jordan constant of absolute normalized norms on $\mathbb{C}^{2}$, Journal of Mathematical Analysis and Applications 244 (2000), no. 2, 515-532.

Weerayuth Nilsrakoo: Department of Mathematics, Khon Kaen University, Khon Kaen 40002, Thailand

Current address: Department of Mathematics, Statistics and Computer, Ubon Rajathanee University, Ubon Ratchathani 34190, Thailand

E-mail address: nweerayuth@sci.ubu.ac.th

Satit Saejung: Department of Mathematics, Khon Kaen University, Khon Kaen 40002, Thailand E-mail address: saejung@kku.ac.th 